\title{
DIVISION IX / COMMISSION 9 / WORKING GROUP DETECTORS
}

\author{
CHAIR \\ MEMBERS
}

\author{
Timothy M. C. Abbott \\ Dennis Crabtree, Craig D. Mackay, \\ Christopher G. Tinney
}

\section{PROCEEDINGS BUSINESS MEETING on 18 August 2006}

\section{Optical detectors}

The same general claims hold true as in the last report and the field is as busy as ever. Community focus is on the deployment of large mosaics (up to $10^{8}$ pixels) to telescopes, the pursuit of programs to build next generation mosaics (of order $10^{9}$ pixels), and the investigation of novel variations in CCD design.

The standard mosaic tile format is still 2048 pixels wide by 4096 or 4608 pixels tall, with pixels either 15 or $13.5 \mu \mathrm{m}$ square. Production is dominated by e2v technologies, although Fairchild Imaging show promise with catalog devices up to $4 \mathrm{k} \times 4 \mathrm{k}$ pixels. The MIT Lincoln Labs consortium is winding down its best effort program after experiencing mixed results. Other small-scale best-effort programs continue, prominent among them being Lawrence-Berkley Laboratory's production of devices for DECam and SNAP.

Conventional CCD performance has stabilized with readout rates of $10^{6}$ pixels/sec or faster with readout noise less than 10 electrons per pixel; typical readout noise floors at slower speeds have been pushed below 2 electrons per pixel, although typically they are around 3 electrons per pixel, and full well capacities are around $10^{5}$ electrons. Backside thinning technologies result in typical responses of over $80 \%$ QE spanning wavebands $200-300 \mathrm{~nm}$ wide.

The demands of large mosaic production translate into tight specifications for detector manufacturers and particularly for consistent device properties in large batches. Flat-field uniformity of a few percent and surface flatness of a few tens of microns are both sought-after characteristics. Charge transfer inefficiency of better than $10^{-5}$ is routine and dark current is neglible below $-100^{\circ} \mathrm{C}$ for most applications.

The first wave of large mosaics was comprised of some twelve cameras containing $8192 \times 8192$ pixels now in place at various observatories around the world. A second wave is already well under way with Megaprime at CFHT and Megacam at MMT in operation for some time and ESO's Omegacam due to begin operations in 2007. A third wave is building with cameras planned and under development, including the Dark Energy Survey's DECam (0.5 Gpixel), WYNN/ODI (1 Gpixel), LSST (3 Gpixel), Pan-STARRS $(4 \times 1.4$ Gpixel), NASA's SNAP (1 Gpixel) and ESA's GAIA (1 Gpixel); the latter two are space-based systems. HETDEX/VIRUS at McDonald Observatory deserves mention for its plans to incorporate 145 spectrographs.

The limited space and massively parallel architecture inherent in these systems mean that controller development is tending towards custom systems for each camera. Nevertheless, NOAO has established the collaborative, open source controller program, Monsoon. Turnkey, off-theshelf CCD systems are closing the gap between amateur and professional demands and such systems are increasingly in evidence at observatories for the less challanging tasks.

Best-effort, wafer-run detector production programs concentrate on the development of novel device architectures or technologies. In particular, worthy of mention are the orthogonal transfer CCD from MIT, Lincoln Labs, USA, to be used in WYNN QUOTA and ODI and PanSTARRS; high-resisitivity, fully depleted CCDs from Lawrence-Berkley National Laboratory, USA to be used in DECam and SNAP; and the L3CCD photon-counting readout architecture from e2v technologues, UK. The development of high speed sensors for active optics systems is also a busy sub-genre. 
CMOS detectors are receiving some attention, with various hybrid designs showing promise, perhaps bridging the optical/near-IR divide, but are not yet serious contenders at the observatory besides their use as multiplexers for infrared detectors.

\section{Infrared detectors}

Teledyne Imaging Systems (previously Rockwell Scientific Company) and Raytheon Vision Systems remain the primary players in the manufacture of infrared detectors. Teledyne continues the development of its HAWAII series of detectors, now working on devices as large as $6 \mathrm{k} \times 6 \mathrm{k}$ with $10 \mu \mathrm{m}$ pixels and $4 \mathrm{k} \times 4 \mathrm{k}$ with $15 \mu \mathrm{m}$ pixels. Several observatories are already operating mosaics of 4 HAWAII-2 arrays with more in development. The UK's VISTA telescope will sport a camera containing 16 of Raytheon's VIRGO arrays. Ever larger focal planes are being developed for space-based systems, in particular SNAP which will fly as many as $362 \mathrm{k} \times 2 \mathrm{k}$ IR detectors.

Raytheon are also moving mid-IR detectors into the megapixel range with the $1 \mathrm{k} \times 1 \mathrm{k}$ Aquarius Si:As IBC array.

Historically, infrared detector development has driven hybrid technologies in the pursuit of higher senstivities over broader wavebands and of Readout Integrated Circuits for faster and more flexible readout schemes and lower power consumption. This trend continues and there are signs of transfer of these technologies to CCD and other visible detector development.

\section{Community interaction}

No less than three periodic conferences offer venues for community interaction, of which the 3yearly workshops "Scientific Detectors for Astronomy" continue to form the core. Most recently, they were held on Hawaii (2002) and on Sicily (2005). The proceedings from the 2005 conference include three overview papers providing comprehensive coverage of the state of existing and planned instruments and detectors at ground based observatories (Simons et al. 2005), CCD technology (Burke, Jorden and $\mathrm{Vu}, 2005$ ) and CMOS detector technology (Hoffman, Loose and Suntharalingham, 2005). SPIE conferences every two years are additional opportunities for community discussion; most recently, they have been at Glasgow, UK in 2004, and Orlando, Florida, USA in 2006. The International Conference on Scientific Optical Imaging has been held on Cozumel, Mexico in 2003 and 2006. The proceedings of all of these conferences provide excellent road maps for following ongoing developments in astronomical detector technology.

The CCD-world mailing list continues to serve the community's day-to-day needs for exchanging news and information on all aspects of detector development. Now hosted at CTIO, its web pages and archives may be found at <http://www.ctio.noao.edu/CCD-world/>.

The Working Group will continue as a Division IX Working Group.

Timothy M. C. Abbott chair of the Working Group

\section{References}

Simons, D. A., Amico, P., Baade, D., Barden, S., Campbell, R., Finger, G., Gilmore, K., Gredel, R., Hickson, P., Howell, S., Hubin, N., Kaufer, A., Kohley, R., MacQueen, P., Markelov, S., Merrill, M., Miyazaki, S., Nakaya, H., O’Donoghue, D., Oliva, T., Richichi, A., Salmon, D., Schmidt, R., Su, H., Tulloch, S., Vargas, M. M. Wagner, R. M., Wiecha, Ol, Ye, B. 2005, in: J. E. Beleteic, J. W. Beletic, P. Amico (eds.), Scientific Detectors for Astronomy, 2005, Proc. Workshop, Taormina, Sicily, 19-25 June 2005, (Springer) p. 13

Burke, B., Jorden, P., Vu, P. 2005, in: J. E. Beleteic, J. W. Beletic, P. Amico (eds.), Scientific Detectors for Astronomy, 2005, Proc. Workshop, Taormina, Sicily, 19-25 June 2005, (Springer) p. 225

Hoffman, A., Loose, M., Suntharalingam, V. 2005, in: J. E. Beleteic, J. W. Beletic, P. Amico (eds.), Scientific Detectors for Astronomy, 2005, Proc. Workshop, Taormina, Sicily, 19-25 June 2005, (Springer) p. 225 